\title{
Sentinel-Meldesystem in der Schweiz?
}

\author{
H. Flückiger
}

Aus der fakultären Instanz für Allnemeinmedizin (FIAM), Universität Bern, und der Arbeitsgruppe Forschung der Schweizerischen Gesellschaft fur All gemeinmedizin (SGAM).

Als die ersten Nachrichten Uber ein "Waldsterben" in der Schweizer Presse erschienen, ist es wohl manchem Arzt ähnlich ergangen wie mir: Ich fragte mich, ob wir als Aerzte eine Morbiditatsanderung in unserer Gesellschaft, die dem "Waldsterben" veraleichbar wäre, rechtzeitic erfassen und auch richtio interpretieren wurden. Wir haben alle miterlebt, wie im Bereiche der Forstwirtschaft Stabstelien neschaffen und ein Erfassungsnetz aufgebaut werden mussten, um erst einmal eine Uebersicht Uber den Schaden zu erhalten. Dass wohl das "Waldsterben" nicht ein isoliertes, auf Baume beschränktes, Geschehen im "Biotop Erde" ist, dïrte jedem, der sich nur einigermassen Gedanken liber die Vernetzung der Lebensvornänge aller Individuen gemacht hat, klar sein. Eine Möglichkeit, sich an verantwortlicher Stelle Uber die Morbiditatssituation beim Menschen, zum mindesten auf der Stufe des Patienten, der den Arzt aufsucht, zu machen, ist das von A. Stroobant in diesem Heft vorgestellte Meldesystem, das in Belgien seit 1978 und in den $N$ iederlanden seit 1970 annewandt wird. Man muss sich natuirlich dabej im klaren sein, dass wir mit einem solchen Meldesystem nur diejenigen Gesundheitsstörungen erfassen, die den Patienten zum Arzt fuhren, wir kennen also auch hier nur die spitze eines Eisberges. Zudem ist es in der Praxis kaum mönlich, die nanze Morbidität festzustellen, wie es in einem Projekt des Oesterreichischen Bundesinstitutes für Gesundheitswesen (1) gewlinscht wird. Wir können, um die Meldestellen (Sentinels-Sentinelles$V$ iajes-Sentinella-Peilstationen-Meldepraxen und wie sie noch genannt werden) nicht zu uberlasten, immer nur einige Merkmale (Krankheiten oder Geschehnisse) registrieren, wie das auch von Stroobandt in seiner Arbeit darcestellt wird. Immerhin wurde uns der Aufbau eines Me1denetzes im jetzigen Zeitpunkt die Struktur zur Beobachtung wichtig scheinender Ereionisse liefern. Nachdem die Arbeits iruppe "Forschuna" der Schweizerischen Gesell schaft fur All nemeinmedizin (2) und die Fakultäre Institution fur Allgemeinmedizin der Universität Bern (FIAM) eine Pilotstudie zur Einfuhrung eines "Sentinel"Netzes- vorerst im Bereiche der Universität Bern- geplant hatten, hat sich auch das Bundesant fur Gesundheitswesen für dieses Projekt interessiert und es wurde eine Arbeits gruppe "Sentinels" eingesetzt, welche nun die Einfuhrung dieses Meldenetzes pruft. Das ursprung1 iche Projekt der FIAM wird nun ab November 1984 als Pilotstudie anlaufen, so dass fur ein auf schweizerischer Basis zu realisierendes Netz Erfahrungen gesammelt werden können. Es ist beabsichtigt, zu Beginn nur vier Krankheiten bzw. Ereignisse melden zu lassen, das sind: Der grippale Infekt, die Pollinosis, Unfälie im Haushalt und die Masern. Beim grippalen Infekt, der naturlich noch näher definiert werden muss, beabsichticgen wir, bei recionalen Häufungen auch virolonische Untersuchungen vornehmen zu lassen. Dadurch sind wir in der Lace, den angeschlossenen Aerzten in einem "feed-back", der renelmassin stattfinden muss, auch fur die Therapie relevante Annaben machen zu können. Dank der Mitarbeit des Bundesamtes fur Gesundheitswesen hoffen wir, diese Rückmelduncen auch zeitrerecht lieférn zu können.

Es entspricht dem Aufbau unseres Gesundheitswesens, dass wir versuchen, die Melde?raxen auf freiwilliner Basis zu newinnen. Der mitarbeitende Arzt soll an den Ereinnissen interessiert werden, um die lotivation des einzelnen zu verbessern. Ich nlaube, dass wir nur auf diese Veise zuverlässine Meldunnen erhalten, die dann auch nütine Rückschlusse erlauben und deren Auswertung rechtfertigen. Es scheintmir ein cangbarer Hen zu sein, um Geschehnisse am ambulant ärztlich versoraten Patienten zu erfassen und mit epidemiolonischen Methoden zu analysieren.

\section{Zusamenfassunn:}

Sinn und Zweck der Einführuna eines "Sentinel-" Meldesystems in der Schweiz werden daraestellt und das Vorgehen zu dessen Aufbau kurz neschildert.

Enregistrement de données de morbiditö par un resseau de medecins en suisse.

L'idêe et le sens d'un système d'enregistrement de la morbidite par des "Sentinelles" en Sujsse est decrit. Les préparatifs de mise sur pied d'un tel système sont en cours.

\section{Literatur:}

(1) Ertler, W et al: Meldepraxen, Studien uber Nützlichkeit und Möglichkeit der Einfuhrung van Meldepraxen in Oesterreich. Wien: Desterreichisches Bundesinstitut fur Gesundheitswesen, 1981 .

(2) Fluckiger H., Forschung in der Schweiz, Allg. med. int. 1983, 12: 186-187.

Adresse des Autors: Lindenstrasse 7 , 3550 Langnau $i / E$ 